\title{
Coulisses
}

Revue de théâtre

12 | Printemps 1995

Varia

\section{Partage de midi : écriture autobiographique}

\section{Antoinette Weber-Caflisch}

\section{OpenEdition}

Journals

Édition électronique

URL : http://journals.openedition.org/coulisses/3283

DOI : $10.4000 /$ coulisses.3283

ISSN : 2546-9460

\section{Éditeur}

Presses universitaires de Franche-Comté

\section{Édition imprimée}

Date de publication : 1 mai 1995

Pagination : 19-29

ISSN : 1150-594X

\section{Référence électronique}

Antoinette Weber-Caflisch, "Partage de midi : écriture autobiographique », Coulisses [En ligne], 12 I

Printemps 1995, mis en ligne le 15 mars 2019, consulté le 30 octobre 2019. URL : http://

journals.openedition.org/coulisses/3283 ; DOI : 10.4000/coulisses.3283

Ce document a été généré automatiquement le 30 octobre 2019.

Coulisses 


\title{
Partage de midi : écriture autobiographique
}

\author{
Antoinette Weber-Caflisch
}

1 Cet exposé ne se propose pas d'interroger - comme son titre pourrait peut-être le faire accroire - les événements présentés dans Partage de midi à la lumière de ceux qui ont effectivement eu lieu dans la vie du poète et dramaturge.

Il s'agira d'examiner la relation que le Sujet de l'écriture entretient avec son texte, en partant de l'idée que cette relation est dominée par un projet de type autobiographique. Cette hypothèse entraînera deux ensembles de questions. On se demandera tout d'abord quel sens et quelle forme un théâtre à vocation autobiographique peut prendre, puisqu'on sait que, par sa définition même, l'autobiographie est un récit et qu'elle engage un mouvement de rétrospection, quand l'action dramatique, elle, se développe tout entière dans le moment présent. On examinera ensuite comment le projet autobiographique est affecté par le fait que Partage se construit en s'étayant largement sur des éléments fictionnels ou plutôt nettement fantasmatiques fortement chargés de valeur symbolique (intégrant, par exemple, contre la logique d'un tel projet, la mort du Sujet). Se pourrait-il que les enjeux du Sujet (son exigence de sens, son désir de réconciliation), enjeux qui sont en profondeur ceux d'une écriture réellement autobiographique, y aient été eux-mêmes soumis à dramatisation, et qu'ainsi la nécessité de transmettre le vécu fidèlement et de façon réaliste ait passé au second plan?

En s'efforçant d'établir la qualité autobiographique de Partage de midi, mon propos devra donc aller au-devant de deux catégories d'objections qui pourront paraitre les unes et les autres insurmontables, les premières étant d'ordre formel, les secondes se rattachant au contrat de vérité que l'autobiographe passe avec son lecteur et avec luimême.

2 Voyons tout d'abord les raisons de forme qui s'opposent à ce qu'on considère Partage de midi comme une autobiographie.

La première de ces raisons, qui est essentielle, tient à la définition même de ce genre. En effet, l'autobiographie se détermine comme le composé interactif de récit d'histoire 
et de commentaire (Weinrich) qu'articule entre eux

l'instance d'un Sujet trinitaire : héros-narrateur-auteur. A quoi s'ajoute un contrat de vérité-sincérité que l'auteur passe avec son lecteur : pour qu'il y ait autobiographie, il faut que l'auteur remplisse des "conditions d'ordre éthique et relationnel » (Jean Starobinski 1970), soit, en d'autres termes qui seront ceux de Philippe Lejeune (1975), qu'il passe avec son lecteur un "pacte autobiographique » : l'auteur doit quelque part (page de titre, préface, incipit, etc.) annoncer à son lecteur la nature de son projet, autrement dit s'engager à fournir l'histoire de sa propre vie. Ainsi seulement l'autobiographie se distingue-t-elle de la fiction autobiographique, c'est-à-dire d'un texte qui affecterait de respecter les règles narratologiques du genre, sans en tenir les enjeux (comme, par exemple, La Vie de Marianne). Il s'agit en somme d'une véritable déclaration d'état civil ! L'auteur affirme être tout à la fois... l'auteur-le-narrateur-lehéros de l'histoire qu'il raconte. Cette conception trinitaire peut se décliner aussi : nom propre (l'auteur), "Je» (le narrateur), Ego (le héros). Presque aucune de ces déterminations ne convient au drame de Claudel, et tout d'abord à l'évidence parce que Partage de midi n'est pas un récit. On y chercherait donc en vain la deuxième Personne de la trinité autobiographique : le narrateur.

Cependant, on pourrait avancer l'opinion ingénue que si la critique a défini l'autobiographie comme récit, ce pourrait être tout simplement parce qu'elle n'aurait pas pensé à regarder du côté du théâtre, les textes canoniques se présentant en effet tous sous la forme de récits. (S'agit-il d'un hasard? On observera en tout cas que les pièces qui, telle La dernière Bande de Beckett, pourraient passer pour autobiographiques, sont extrêmement rares. Quant aux Essais de Montaigne, dès lors que l'autobiographie a été déterminée formellement par le récit, on a cessé de les ranger dans le cercle des textes autobiographiques où on les a fait figurer tout d'abord.) Mais, en ce qui concerne Partage de midi, l'élément formel n'est pas seul en cause: en fait, Claudel semble redistribuer à sa façon toutes les composantes d'une forme sur laquelle la critique récente s'est au contraire efforcée de statuer d'une manière qu'on pourra trouver un peu légaliste, peut-être, mais qui a le mérite de clarifier ce qui est en cause.

Commençons par remarquer que, si, comme nous le verrons, Claudel a introduit dans Partage de midi de nombreux éléments fictionnels (ne serait-ce que la " mort annoncée » du mari de la belle Ysé qui n'a pas de répondant référentiel), il a pourtant contracté avec son lecteur un pacte autobiographique, et cela de manière fort classique. Dans la Préface de son drame (présentant la version de 1948), l'auteur affirme à propos de son personnage Mesa :

Et comment se serait-il fait que sur ce bateau une femme à la fin ne l'attendît pas? [...] Il n'est plus question de Dieu pour le moment, mais voici en face de moi, maintenue et sans que je puisse m'y soustraire, cette image de Dieu qui a levé les yeux sur moi. (p. 46)

Nous reconnaissons dans ce glissement du «il » au « je » un élément déterminant qui contraint le lecteur à prendre en considération la vocation autobiographique de Partage de midi. Argument majeur, mais non pas décisif cependant. En effet, propre à la forme théâtrale, l'absence du narrateur suppose celle de l'angle de vue unificateur du Sujet de l'écriture. Or cet angle de vue se rattache à la conception même de l'autobiographie. Au théâtre, quand il s'agit d'un drame et non seulement d'un soliloque, nous n'avons pas directement affaire à une conscience ordonnatrice, à un Sujet qui réélabore son expérience, qui « somme » tous les éléments d'une vie, mais nous voyons débattre et se débattre des Sujets, et il advient toujours un moment où la scène confronte des 
consciences, affronte des volontés, des projets autonomes aux prises dans le moment présent. Le théâtre semble donc bel et bien remplacer le regard rétrospectif et la conscience unificatrice de l'autobiographe par ce qui se situe à leur opposé. S'agissant de textes théâtraux, je proposerai donc de parler, plutôt que d'autobiographie, d' écriture autobiographique. Nous laisserons donc de côté les contraintes d'un genre lié structurellement à la forme du récit, pour interroger les enjeux d'un projet : comment au théâtre le Sujet s'y prend-il pour placer sa vie sous son propre regard et pour élucider l'énigme de sa propre destinée ?

Admettons qu'une écriture autobiographique est à l'œuvre chaque fois qu'un Sujet situe son passé, mais aussi son vécu actuel, dans une perspective critique dynamique, c'est-à-dire réflexive et exploratrice. La formule qui convient au récit autobiographique - Voici celui que je fus, comment je suis devenu, qui je suis - se transformera donc tout naturellement dès lors qu'il s'agit de théâtre: un auteur expose (et non raconte) comment "Je" deviens celui qu'Ego aura été. Toute l'action du drame a donc pour seul enjeu la constitution d'un Sujet.

Ce Sujet, nous avons vu que Claudel l'identifie dans sa Préface. Mais, dans l'ensemble, tel qu'il le présente, le Mesa en qui il se reconnaît est avant tout Ego, le personnage confronté à la nécessité d'opérer un " partage » : « Le moment est venu, en ce milieu de la vie, de la proposition centrale qui ne saurait être éludée.» (p. 46) Il nous reste à découvrir comment le texte dramatique constitue ce personnage de façon qu'il ne puisse être dissocié de sa fonction de Sujet (qui est d'ordonner le vécu, de donner un sens à la confusion de la vie). Ceci évidemment est autrement plus difficile que d'assurer un report biographique.

4 Ne se bornant pas aux monologues réflexifs ou aux soliloques d'une conscience affrontée à son passé, Partage de midi va loin dans l'inscription du projet autobiographique dans la forme théâtrale. Mesa n'est pas qu'une voix montée sur scène, un "Je » rivé à sa voix. Certes, Mesa ( $\mathrm{Je}$ ») est une voix (souvent une voix lyrique), mais c'est aussi un personnage (Ego), un être opaque aux autres et à lui-même. Pour comprendre vraiment comment Partage inscrit le projet autobiographique dans la forme théâtrale, il faudra donc interroger la relation Mesa-Ysé.

Dans les premiers duos d'amour («Mesa, je suis Ysé, c'est moi »), Ysé tout d'abord apparaît complètement en elle-même, pour elle-même - c'est alors un Sujet -, ce qui la rend si séduisante à nos yeux. Mesa, quant à lui, présente à ce moment avant tout son rôle d'Ego. De même, semble-t-il au premier abord, dans les moments où se trame la liquidation (toute fictionnelle) du mari, l'entente apparente des amants n'incite pas à penser que le texte se situe dans la problématique d'Ego et l'angle de vue de «Je ». On croit voir simplement se dérouler l'histoire d'un couple aux prises avec les problèmes de l'adultère. Cependant, dans la forme énigmatique qu'elle prend, la tentative d'Ysé de mettre en garde son mari peut nous faire comprendre que le regard qu'elle jette sur le projet criminel n'est pas le même que celui de son amant. Cependant, sans adhérer complètement ni s'opposer à celui de Mesa, son désir ambivalent n'accédera pas à une formalisation nette. Son point de vue reste en fait dans l'ombre. Il ne devrait donc pas nous paraître autrement surprenant que, confrontés en apparence à du biographique, nous découvrions que ce biographique est situé dans la perspective autobiographique qui est celle de Mesa-« Je ». Mais alors, d'où lui vient donc ce curieux statut de fiction? Nous le comprendrons si nous intégrons cette fiction (pseudo-biographique) au sein du projet autobiographique général, car cela nous amènera à constater un fait d'importance : la rencontre d'Ego (Mesa) et de son objet (Ysé) introduit au monde du 
fantasme: le fantasme œdipien de Mesa: se débarrasser du rival. On peut donc considérer que le deuxième acte de Partage accrédite ouvertement et de façon évidente, l'idée toute moderne que la représentation du fantasme est la voie qui conduit au monde du Sujet. Que les critiques aient pu montrer que ce fantasme se formule par le biais d'un emprunt à l'histoire du roi David devient dans cette optique éminemment intéressant : l'importance du personnage historique auquel il est fait référence et tout à la fois l'origine de l'intertexte (les livres sacrés) accusent le coloris mégalomaneinfantile du fantasme.

Le ton change ensuite. Au dernier acte, Mesa assume clairement et complètement sa position de Sujet autobiographique réflexif et douloureux. Nous n'examinerons pas sur ce point le "Cantique» de Mesa, qui rappelle de trop près le dialogue familier et confiant avec son Créateur de l'Augustin des Confessions pour qu'il soit absolument probant de s'y attacher (la démonstration serait trop facile), mais nous nous attacherons à sa relation avec Ysé.

Mesa obtient progressivement qu'Ysé, cette femme aux allures si libres tout d'abord et qui aime tant à rire, entre dans le rôle poignant qu'il lui fait jouer. On remarquera en effet qu'à partir du moment où elle lui a cédé, elle a en sa présence un tout autre caractère, un tout autre tonus, et qu'elle accède à un tout autre langage, en un mot qu'elle n'est plus la même personne quand elle est avec lui.

Le premier de ces rôles est celui de la femme interdite. Si l'on est autorisé à croire qu'il s'agit bien là d'un rôle, c'est qu'Amalric a clairement expliqué à Mesa ce qu'il en était en réalité (c'est-à-dire tout le contraire) et qu'Ysé elle-même se plaint du peu de cas que Ciz fait de son état de mari: alors qu'il devrait la "défendre» (contre les entreprises des galants) et « lui défendre " (l'adultère), il s'en va et la laisse quasiment au pouvoir de Mesa. Si donc malgré une insistance si nette, Mesa considère en Ysé la "femme interdite", c'est que nous devons comprendre qu'il la veut telle. C'est ainsi en dépit de la réalité évidente (à laquelle le spectateur a accès grâce aux scènes qui font dialoguer Ysé et Amalric), mais aussi dans le déni de ce que Mesa constate lui-même, qu'une Ysé, en réalité métamorphosée par le prisme du désir de Mesa, peut être située dans la perspective et montrée dans l'angle de vue du Sujet. On ne verra donc pas dans la complaisance dont fait preuve Ysé en jouant les rôles que lui destine le Sujet un fait biographique : comprenons bien plutôt qu'ainsi seulement peut s'établir au théâtre la présence ordonnatrice et rayonnante d'un Sujet autobiographique. Introduisant une incohérence stylistique qui doit surprendre, l'écart entre l'Ysé réelle et l'Ysé-de-Mesa est ce qui rend visible le point de vue autobiographique. L'importance de la déviance par rapport au réel qu'impose ce point de vue se mesure au contraste si vifs qu'entretiennent entre eux les deux registres où se manifeste la présence d'Ysé : celui de la comédienne et celui de la tragédienne.

Mais il y a plus: Ysé est bientôt présentée comme une bonne élève. A certaines remarques de Mesa - qui, par exemple, critique son rire ("On ne rit pas aux éclats", p. 84) -, elle comprend intuitivement ce qu'il attend d'elle. C'est bien un rôle qu'il lui faut apprendre. Nous assisterons à son apprentissage :

Mesa : Je sais que je ne vous plais point.

Ysé : Ce n'est point cela, mais je ne vous comprends pas,

Qui vous êtes, qui ce que vous voulez, qui

Ce qu'il faut être, comment il faut que je me fasse avec vous. (p. 76)

Ysé : Pourquoi est-ce que j'ai dit cela tout à l'heure?

Je n'en sais rien, Je ne sais ce qui m'a pris tout à coup,

Quelque chose de tout nouveau, 
Qui m'a poussée. A peine dit

Le mot, j'en ai été choquée, (pp. 80-81)

5 A partir du moment où Ysé comprend ce qu'il en est, elle s'exécute en actrice de son rôle - et alors, bien sûr, la comédienne réelle devra surjouer, comme le signale l'ironie de sa répartie (le calembour sur le mot « défendue ») :

Ysé : Je suis à toi,

Je ne me recule pas, je te laisse faire ce que tu veux.

Mesa : O Ysé, c'est une chose défendue.

Ysé : Est-il vrai?

[...] Pauvre Ysé ! je ne la croyais pas si défendue ! (pp. 105-106)

Dans cette dernière réplique, nous pouvons prendre la juste mesure de ce qui sépare l'Ysé réelle de son image dans la perspective de Mesa. En effet, dans son discours, Ysé distingue encore son être et son rôle, comme le montre le fait qu'elle parle d'elle-même à la troisième personne: "Je ne la croyais pas...". Mais bientôt, du moins en sa présence, elle se laissera entièrement assimiler aux diverses conceptions que Mesa a d'elle: amoureuse fatale, divinité naturelle, épouse spirituelle, et même âme morte bientôt destinée à errer au ciel telle une étoile (c'est le sens que l'on peut donner à son retour fantomatique auprès de Mesa mourant et au dernier duo du drame). A la fois maîtresse coupable, amante mythique et mystique, femme " interdite ", "impossible ", elle rejoint finalement l'image de la mère et l'en-deçà de la vie que celle-ci évoque : « ce corps lourd, dira Mesa en lui parlant d'elle, qui est ma mère et ma sœur et ma femme et mon origine » (p. 149). Et déjà " Comment est-ce qu'il faut vous appeler ! Une mère. [...] Et une sœur, et je tiens votre bras rond et féminin entre mes doigts » (p. 106). Le corps d'Ysé, Ysé elle-même, entrent ici dans une série d'appropriations métaphoriques qui la métamorphosent en image dans la conscience de Mesa. Ces métaphores sont la trace, présente au sein de l'écriture, du phénomène que nous analysons ici. La transformation du Sujet-Ysé ( “ C'est moi, Ysé... ») en métaphore vivante est ce qui permet de marquer textuellement la belle Polonaise au coin de la subjectivité de Mesa, de l'inféoder à son point de vue en la transformant en être de langage. Si bien évidemment, il n'accède pas à la charge qui est celle du narrateur dans le récit autobiographique, Mesa ainsi devient pourtant instance textualisante. Le spectateur-lecteur s'en rend d'autant mieux compte qu'en présence des deux autres hommes, Ysé reste la femme réelle et « habituelle » qu'elle a toujours été. Le jeu de scène marque nettement cela: à Ciz, elle demande d'apporter sa « chaise » (p. 58) comme, à l'autre bout du drame, elle sera pour Amalric celle qui tout bonnement fait du thé (p. 125).

7 Mais il ne s'agit pas seulement de nous faire regarder Ysé par les yeux de «Je »-Mesa et ainsi de faire apparaître le point focal d'où toute l'histoire des deux amants est vue. Il importe de voir que, dans la conscience de Mesa, la métaphorisation d'Ysé joue aussi un rôle et qu'elle y correspond aussi à une fonction dramatique. A la fin du premier acte, Ego-Mesa commence à comprendre qu'il ne peut pas se contenter d'imaginer ce qu'il veut savoir ou croire des autres et de lui-même. L'amour le fait sortir de sa bouderie et l'adresse à l'Autre. Ce qu'Ego demande profondément à Ysé, c'est qu'en jouant son rôle de métaphore de la femme, elle l'aide à modifier l'image de la femme qui est la sienne, ou sans doute plutôt à la déplacer, et, à partir de là, qu'elle lui permette d'engager une nouvelle relation à l'Autre. Cet aspect des choses sera fortement accentué dans les versions tardives.

C'est bien dans le vœu d'une telle transformation d'Ego qu'il faut trouver la réponse à la demande de sens de « Je »: 
Pourquoi?

Pourquoi est-ce que cela arrive ? Et pourquoi faut-il que je vous rencontre ? (p. 77)

Pourquoi cette femme? pourquoi la femme tout d'un coup sur ce bateau?

Qu'est-ce qu'elle s'en vient faire avec nous? (p. 142)

Or le questionnement et la découverte du sens de sa vie (comme, dans le cas de Claudel, l'imposition d'un sens à sa souffrance) sont précisément ce qui constitue en Sujet le scripteur autobiographique. Dans le point de vue rétrospectif qui est celui d'un Claudel autobiographe, tout s'explique, car tout explique. Nous voyons qu'ici le projet autobiographique se réalise dans la forme du théâtre dans la mesure où il rencontre l'idée toute claudélienne que ce qui existe comme ce qui arrive dans la vie a un sens: un sens qui doit certes être découvert, mais aussi - précisément en vue de cette découverte - constitué par le Sujet. Au narrateur du récit autobiographique peut donc se substituer un personnage-auteur qui déchiffre ce qui arrive dans sa vie comme s'il s'agissait de lire les lignes d'un texte, et qui, en le lisant, constitue ce texte, en ceci qu'il confère une existence à ce qui déjà a sens : « les mots ont besoin de notre voix », lira-ton dans Le Soulier de satin (II, 5). Nous comprenons maintenant la nécessité qui s'impose de façon si forte à Mesa de développer une énonciation poétique, lyrique (Voir, bien avant le "Cantique de Mesa », le final du premier Acte).

Tout en continuant de s'attacher au personnage de Mesa, notre lecture va maintenant prendre en compte les thèmes que développe l'œuvre.

Ici, un nouveau scrupule surgit : peut-on réellement parler d'écriture autobiographique dès lors que seuls quelques moments clés de la vie sont traités? A elle seule, une crise peut-elle réellement permettre de thématiser un enjeu autobiographique? De plus, peut-on admettre que ce moment se trouve situé loin de ce qui, sur le modèle des Confessions, a toujours été traité comme le seul véritable point-origine, c'est-à-dire les scènes de l'enfance? La spécificité autobiographique ne se définit-elle pas en fin de compte par l'ambition de reconstituer (ou de constituer) la genèse d'une conscience?

Faisons ici un bref rappel. Comme le montrent, par exemple, les épisodes hautement symboliques de fruits volés tant chez saint Augustin que chez Rousseau, l'insistance sur les scènes d'enfance, agréables ou traumatisantes, picaresques ou tragiques, a entraîné la création d'un véritable topos. Anticipant les intuitions de Freud, 1'autobiographe fait de l'enfance du Sujet une sorte de noyau originel qu'il constitue en puissance de sens. Autrement dit, il investit l'enfance d'un pouvoir herméneutique autonome qui lui permet de mettre en mouvement la «machine » autobiographe. Dans les Confessions de Rousseau, la fessée Lambercier, l'épisode "carnifex», etc., sont emblématiques à cet égard. Pour le Sujet, il s'agit d'événements véritablement originels, puisqu'ils vont lui servir à ou lui permettre de formater rétrospectivement la destinée de ses pulsions, la tendance de son caractère, etc.

Certes, il n'y a pas d'enfance dans Partage. Cependant on voit bien que Claudel y présente une scène originaire qu'il investit du même rôle symbolique que les grands textes autobiographiques impartissent aux mythiques récits d'enfance. L'échec de la profession monastique vaut, pour Mesa, sinon une naissance, du moins un récit d'enfance.

Saisissons l'occasion de remarquer (non sans étonnement) que cette scène originaire n'est pas celle qu'on aurait pu attendre, connaissant la biographie du poète. En effet, Claudel ne convoque pas la célèbre conversion de 1886, qui fut pourtant le berceau de toute l'œuvre à venir. Il ne la mentionne même pas, proposant au contraire comme point-origine le moment où Ego a été «repoussé » par Dieu alors qu'il avait fait 
(pensait-il) le sacrifice de ses intérêts propres pour entrer dans les ordres. Ce rejet, la pièce le présente à la fois comme une blessure cruelle et un affront incompréhensible : comme une rupture indépassable.

Il faut comprendre que, tout en restant totalement aveugle sur les tenants et les aboutissants de son choix existentiel erroné, Ego est sommé d'admettre que sa vocation religieuse a été reçue comme une fausse vocation. Or il n'en est pas capable. Le moment-origine que propose Partage est donc celui du rejet, mais c'est en même temps celui de l'aveuglement et de l'impossible reconnaissance de la vérité, car ce qui a été son véritable désir reste ici hors de la prise du Sujet : trouver à la fois un refuge et une solution à ses contradictions.

Situé hors champ, ce moment-origine sera re-présenté au moment où, sur le bateau de l'Acte I, Mesa s'offre à Ysé (« je me donne moi-même ») comme auparavant il rappelle qu'il s'est offert à Dieu (« que pouvais-je faire?»). Il importe à notre propos d'observer que ces errements du Sujet peuvent parfaitement être montrés à la scène sans être modalisés. En effet, ils se désignent d'eux-mêmes; et là où le récit autobiographique produirait un commentaire avisé du narrateur soulignant l'ambiguïté du Sujet qui prétend se donner alors qu'il désire être pris («Prenez vous-même... »), la dénégation suspecte ( «Je n'ai pas été repoussé...»), le coq-à-l'âne révélant une censure (ellipse marquée par le tiret), le discours théâtral, qui utilise les ressources de la rhétorique, laissera à la discrétion de l'acteur ou du lecteur la charge d'interpréter l' « en-dessous » du texte :

Mesa : Que pouvais-je faire ? où est ma faute?

Je suis sommé de donner

En moi-même une chose que je ne connais pas.

Eh bien, voici le tout ensemble ! Je me donne moi-même. Me voici entre vos mains.

Prenez vous-même ce qu'il vous faut.

Ysé : Vous avez été repoussé?

Mesa : Je n'ai pas été repoussé. Je me suis tenu devant Lui Comme devant un homme

qui ne dit rien et qui ne prononce pas un mot.

- Les choses ne vont pas bien à la Chine. On me renvoie ici pour un temps. (p. 79)

La réplique d'Ysé (« Vous avez été repoussé? ?) confirmera cette lecture: Mesa, ici, joue à de nouveaux frais la scène où il s'est offert à Dieu. Conjugué au présent, le verbe " je suis sommé de donner » vaut pour les deux scènes, l'originelle et sa reprise. Bien entendu (comment s'en étonner ?) les deux fois, les entreprises de Mesa auront les mêmes effets. Sa tentative de "se donner » à la femme dont il est amoureux se solde par un échec, un échec qu'Ysé, adroitement et charitablement, l'engagera à assumer : "Convenons que nous ne nous aimerons pas» (p. 83).

Mesa se drape dans sa bouderie mélodramatique chaque fois qu'il évoque ce qu'il présente comme une injustice de Dieu. Il revendique en quelque sorte un noble chagrin : "Cela du moins est à moi. Cela du moins est à moi ", ressasse-t-il à tout moment. La répétition de la formule et son retour sont précisément ce qui doit nous faire comprendre qu'il s'agit d'une affirmation dont le Sujet n'a pas les moyens de dépasser l'erreur qui la sous-tend. Devenant par là même constitutive du Sujet luimême, cette incapacité va s'agréger à la scène-origine qui doit permettre à Mesa d'étalonner le vécu qu'il présentera sur son modèle. Comme le montre une périphrase éclairante, la confrontation avec "cette image de Dieu qui a levé les yeux sur moi" (p. 46), c'est-à-dire la rencontre de la belle Ysé, sera en effet explicitement présentée (et non seulement jouée) comme une seconde version du drame personnel de Mesa: l'échec religieux. 
«Cette image de Dieu qui a levé les yeux sur moi » et non pas "sur qui j'ai levé les yeux » : telle est, encore en 1948, la formule par laquelle Claudel désigne la relation d'Ego avec l'objet de son amour. Il avait alors quatre-vingts ans. Faut-il croire que l'attitude du Sujet victime ou du moins passif - une attitude qui doit entrainer la présomption d'innocence - serait celle du vieux Claudel ? Parvenu au seuil de son grand âge, admet-il, comme son héros, que le pauvre Ego a été séduit et abandonné par la femme aimée, tout comme il l'aurait d'abord été par Dieu? Bien entendu, il n'en est rien. Le nouveau dénouement de Partage, la conclusion du Soulier de satin montrent assez que l'indépassable aura pu être dépassé. Simplement, 1'écriture autobiographique théâtrale ouvre à la pratique du jeu de rôles : le temps de relire et d'évoquer une œuvre de jeunesse pour l'expliquer à la postérité, « Je » redeviens celui que Ego a été, un Sujet qui n'était pas parvenu à l'élucidation de son destin. Sur un point, en tout cas, Claudel n'a pas varié : ce Sujet à qui la passion amoureuse paraît une expérience seconde ne s'étonnera pas qu'elle tire sa lumière de l'expérience religieuse dont il confirme le caractère premier, ni d'ailleurs (nous allons y venir) que la seconde puisse d'une certaine façon éclairer la première en retour.

Mais il y a sans doute plus à voir qu'une sorte de cellule événementielle originelle dans l'évocation du jeune novice renvoyé rudement à la chine en guerre alors qu'il recherchait en France la paix du monastère : «Les choses ne vont pas bien à la Chine. On me renvoie ici pour un temps » (p. 79). Il faut encore discerner dans le scénario du rejet de Claudel-Mesa une origine qui serait à l'œuvre non seulement (névrotiquement?) dans la vie et dans le drame comme principe d'organisation de la matière biographique, mais aussi dans l'écriture autobiographique. Cette crise a éveillé avec la volonté de comprendre le besoin d'écrire, et d'écrire sur soi.

Certes, on peut penser que, munie d'un outil herméneutique aussi paradoxal, l'élucidation autobiographique ne pourra que se borner à aller du même au même, ou à créer du même. L'entreprise semble donc limitée par sa méthode même. Pourtant ce qu'une telle méthode permet de dégager est essentiel : l'affect qu'elle réinvestit dans le geste de l'écriture. Comparons : «Je me suis donné, / Et Vous ne m'avez point accepté » (p. 143) et "Je l'aimais [...] et elle m'a fait cela!» (p. 144). C'est bien, de part et d'autre, associé à deux événements découpés au sein du réel au gré d'un pattern unique, le même sentiment qui se dégage, ce sera aussi, comme nous allons le voir, la même présentation de l'histoire.

11 Cependant interpréter le passé de son héros grâce à la mise en place d'un schéma répétitif ne saurait être le dernier mot du dramaturge. Il lui faut une action.

Et c'est un fait qui doit nous ouvrir des horizons que, dans sa Préface tardive de 1948, après avoir suggéré l'idée que le débat entre l'homme et Dieu se rejoue une seconde fois avec la même piteuse issue entre l'homme et la femme, Claudel propose une tout autre explication. Celle-ci correspond, bien entendu, à la nouvelle lecture de sa vie à laquelle il est alors parvenu et dont témoigne à cette époque le final rénové du nouveau Partage. Mais nous verrons qu'elle permet également de donner un sens à l'extravagant final érotico-mystique de la version de 1906 qui, de toute façon, imposait déjà de remettre en question l'accusation de trahison ou de rejet portée à rencontre de la femme.

Dans cette Préface, Claudel avance sa nouvelle interprétation de façon assez cachée, au gré d'une citation biblique qui, présentée hors de son contexte et traduite de façon à paraître obscure au lecteur moderne, a certainement été destinée à rester incompréhensible aux non-initiés. Le dernier paragraphe offre en effet sans aménagements, en guise de conclusion « ouverte », une citation qui rapporte à Osée XI, 
$4:$ : Je te tirerai dans les liens d'Adam, dit le prophète Osée ", ce qui est la traduction claudélienne de "In funiculis Adam traham eos in vinculis charitatis » (Vulgate), verset que la Bible Fillon rend de manière moins sibylline: "Je les ai tirés par des cordes humaines, par les liens de l'amour ».

Seul le rappel du contexte permet de comprendre de quoi il s'agit. En effet, dans Osée, "Je les tirerai par les liens de la chair» répond à "Je les ai appelés et ils se sont éloignés ». Il faut comprendre que c'est parce que les hommes sont sourds à l'appel direct de Dieu, que Dieu, pour les attirer à lui, se sert comme d'un appât de l'amour charnel. Claudel qui traduit «traham eos in funiculis Adam» par «je te tirerai dans les liens d'Adam » propose donc une application du verset biblique à son drame personnel. Ce faisant, il donne une lecture entièrement neuve du dramatique rejet de Dieu et par conséquent de toute la pièce. Il s'agit en fait d'un retournement complet, car ce n'est plus alors Dieu qui refuse le sacrifice de Mesa, c'est Mesa qui s'est éloigné de Dieu et que Dieu "rattrape» par le moyen des «liens de la chair», entendons par l'amour d'Ysé. Cet amour aboutit à son sacrifice : «Ce qui est refusé à la passion, le sacrifice, qui sait si d'une manière ou de l'autre, il ne pourra pas l'obtenir?» Ces mots par quoi s'achève la Préface de 1948 nous permettront enfin de répondre à la question de Mesa : "pourquoi la femme", "pourquoi cette femme? ? Nous devons comprendre que la rencontre «crucifiante » d'Ysé doit s'expliquer au bout du compte non pas comme la répétition de l'échec de Mesa, mais comme sa conséquence. Dans cette lecture, Mesa n'aurait pas été rejeté. Il a fait faux bond: "Je les ai appelés et ils se sont éloignés ». C'est finalement l'amour de la femme poussé jusqu'au sacrifice qui vient à son secours. Le mariage dans la mort réintègre dans l'ordre religieux un Mesa qui, ayant commis l'adultère, peut enfin se trouver coupable et s'offrir enfin « honnêtement » à Dieu :

Mesa : [...] Nous pouvons donc tourner honnêtement le visage vers le Vengeur,

En disant : « Nous voici. Payez-vous sur ce que nous avons. » Nous pouvons cela.

Et

Puisque tu es libre maintenant,

Et qu'en nous près d'être détruits la puissance indestructible

De tous les sacrements en un seul grand par le mystère du consentement

réciproque

Demeure encore, je consens à toi, Ysé !

Voyez, mon Dieu, car ceci est mon corps! (p. 153)

C'est donc parce qu'Ysé a cédé à l'amour, entraînant Mesa dans le péché qu'elle lui donne l'occasion de débloquer une situation qui menaçait de rester sans issue («Je suis parti, il me faut revenir à la même place » p. 79) : felix culpa. L'amour contraint Mesa à sortir de son repli narcissique et, en assumant «honnêtement » sa part du péché, à abandonner son inconsciente mauvaise foi. Ainsi l'événement dont nous avons vu qu'il sert à Mesa de principe interprétatif de sa propre vie, la Préface finit par montrer qu'en fait il fallait qu'il se répète pour que Mesa trouve dans ce second épisode le moyen de renouer la relation rompue avec Dieu dans le premier. Claudel préfacier, qui tout d'abord nous l'avons vu, s'identifiait à Mesa, prend finalement ses distances par rapport à lui, le considérant, grâce à l'intertexte biblique, du point de vue de Dieu. La triade auteur-Je-Ego s'en trouve dissociée. Il s'ensuit que toute l'aventure est reconsidérée sous un jour nouveau. Du point de vue dramatique, il importe de saisir que, dans cette perspective, l'aventure amoureuse expliquerait ou plutôt révélerait la vérité concernant le (soi-disant) refus de Dieu, et non pas l'inverse. C'est en ce retournement de point de vue auquel «les liens d'Adam» ont contraint Mesa que consiste l'action de Partage de midi. Affectant expressément le devenir de la conscience 
du Sujet, ce retournement s'inscrit dans le droit fil de la vocation autobiographique du texte.

En fait, on peut supposer qu'il y a eu dès le début deux scénarios en concurrence. En effet, le verset d'Osée que Claudel cite dans sa Préface de 1948 et que nous venons de lire figure déjà dans la toute première page du Journal, en 1904 !

Il est essentiel pour saisir l'importance des enjeux en cause de savoir qu'au moment où il écrit les premières esquisses de ce qui deviendra Partage de midi, Claudel entreprend de tenir un Journal et qu'il réfléchit alors précisément au problème de la bonne foi qui devrait présider à son double engagement dans l'écriture du Moi. Le 24 février 1905, le jour où il apprend « l'affreuse trahison » (événement qui va lui permettre de trouver la forme du scénario définitif de Partage), il recopie dans son Journal une phrase où saint Augustin expose exactement la difficulté que lui, Claudel, vient à son tour de rencontrer. Le saint patron des autobiographes y réunit sous le terme d'avaritia, défaut dont il s'accuse, et la mauvaise foi du nouveau converti qui prétend ne rien lâcher c'est l'avarice que Claudel attribue à plusieurs reprises à Mesa - et l'avidité de l'ancien païen qui prétend seulement gagner à sa conversion (en latin, «avaritia » a les deux sens d' "avarice» et d' "avidité »). Avec sa grande perspicacité psychologique, Augustin souligne, dans la suite de la phrase que Claudel recopie, le danger de la mauvaise foi qui entraîne à "mentir au point de ne plus savoir soi-même ce qui est vrai ». Comprenons qu'en croyant à son propre mensonge, on entre dans ce qui ne serait qu'un rôle, alors même que ce rôle doit consister à fournir une interprétation ayant statut de vérité. Des questions se profilent: l'autobiographe vise-t-il l'autojustification ou s'attache-t-il à découvrir le vrai? Peut-on atteindre la vérité dès lors qu'on ne veut rien abandonner de ce qu'on a été ? Et surtout ceci : la vérité a-t-elle les moyens de se dévoiler dans l'immanence du projet autobiographique?

Le Journal montre donc que, bien avant la lecture rétrospective de Partage qu'il donne dans la Préface de 1948, lecture qui enchaîne deux principes d'explication successifs, Claudel était préparé par la lecture des Confessions de saint Augustin à remettre en question le principe d'explication de sa destinée qu'il choisit pourtant à cette époque de mettre au premier plan: Mesa séduit et rejeté. Il faut donc s'attendre à trouver les deux principes opposés également dans la première version de ce drame.

Premier principe : le même répète le même. Interpréter consiste alors à mettre en série. L'explication procède à partir d'un noyau-origine irréductible: une séquence traumatisante, dérangeante ou tout simplement rebelle à l'oubli, telle une sorte de péché originel privé, propre au Sujet, se révèle déterminante, mais pour des raisons obscures, puisque son pouvoir reste inexplicable : «J'ai été trouvé manquant. J'ai perdu mon sens et mon propos» (p. 79). Présent dans l'autobiographie moderne (de Rousseau à Leiris...), ce principe est frappé, nous l'avons vu, de cette insuffisance constitutive qui par là même le rendra si parfaitement apte à rendre compte d'un Sujet plongé dans l'immanence de son humanité. Le Sujet qui l'applique ne peut qu'accumuler son vécu, et le moment de la vérité n'est jamais qu'un point de fuite, une épiphanie qui se dérobe parce qu'elle se déplace avec le moment de l'écriture, mais toujours à son horizon.

$\mathrm{Si}$, comme nous l'avons vu, Mesa-Claudel recourt à cette pratique de l'interprétation immanente, il importe de comprendre qu'il n'en reste pas là. Au contraire, la seconde séquence, son caractère de répétition une fois reconnu, donnera au Sujet l'occasion de dépasser son passé. Avec Ysé, en effet, le Sujet assumera ce qu'il avait esquivé avec Dieu : la rencontre, la fusion et même une sorte d'assomption rêvée. Substituée à Dieu, 
Ysé fera de Mesa le parèdre associé à sa divinité symbolique de grande déesse, et Mesa non seulement entrera dans ce rôle, mais il le surmontera.

Dans l'union religieuse telle que la propose le monastère, l'âme chrétienne s'abîme dans l'humilité de sa condition. Mesa tombant aux genoux d'Ysé ne fera tout d'abord pas autre chose. Mais bientôt c'est lui qui, en se saisissant d'Ysé - une Ysé véritablement associée à la nature, puis à quelque divinité stellaire ou lunaire -, c'est lui qui va prendre la divinité «entre <ses> bras» (p.113). Au bout du compte, il la sacrifiera, en ceci semblable au prêtre de Baal évoqué dans la première scène («Ce ne sont plus des baisers, c'est le couteau dans ses entrailles », p. 54). Nous voyons donc qu'un fantasme de toute-puissance peut se greffer sur la séquence amoureuse et donner au Sujet l'occasion de prendre une revanche imaginaire sur la situation qu'elle répète. Le "petit Mesa» (p.154) dont la vocation a été rejetée et dont la déception remplit de ses échos l'acte I est devenu à la fin du III « le grand mâle » (p. 158) qui fonde son « Ordre» (p. 153). Pourtant son triomphe ne consiste pas à dominer une Ysé qu'il entraîne dans sa mort - sans l'associer à son apothéose solaire -, mais bien à retourner le schéma initial et à se trouver en fin de compte plus fort que la divinité ou sa déléguée, la femme, qui l'ont rejeté ou devant qui il a dû s'incliner ou décliner. Sur ce point, la version du premier manuscrit et celle de 1906 sont d'accord: elles finissent toutes deux par une glorification du Sujet qui ne fait que prendre de l'ampleur d'une version à l'autre :

Et voici une sécession dans mon cœur, et je me tourne épouvantablement vers toi, et tu es à moi, et tout m'est égal, et je suis le plus fort ! (p. 203)

[...] tous voiles dissipés, moi-même, la forte flamme fulminante, le grand mâle dans la gloire de Dieu,

L'homme dans la splendeur de l'août, l'Esprit vainqueur dans la transfiguration de Midi! (p. 158)

La version de 1948 déguisera davantage ce que Philippe Lejeune nomme la mythomanie de l'autobiographe. Pourtant il s'agit toujours pour Mesa de conduire Ego à l'apothéose : il cueille le ciel étoilé dans sa main et Ysé «s'enfonce dans la nuit à ses pieds".

Tout cela qui est de l'ordre du fantasme est clairement donné pour tel. Nous constatons à nouveau qu'en pionnier de l'écriture autobiographique moderne, Claudel étend son exploration aux formations de l'imaginaire.

Mais l'autre principe d'interprétation de sa destinée, celui que Claudel pointe en citant le prophète Osée, est également actif dans Partage, même s'il est beaucoup moins visible. Il apparaît négativement dans le creux de la question qui fait retour, « Pourquoi la femme?", question qui (du moins dans la version de 1906) restera sans réponse dans le discours du Sujet, et ceci alors même qu'Ysé lui souffle fort explicitement la solution de son problème. Mesa, cependant, reste sourd à ses suggestions dont il ne comprend ni le sens ni la portée :

Mesa: [...] Pourquoi venez-vous me rechercher? pourquoi venez-vous me déranger?

Ysé : C'est pour cela que les femmes sont faites.

Mesa : [...] Qu'est-ce qu'il y a à attendre, qu'est-ce qu'il y a à comprendre chez une

femme? (p. 75)

Ysé : Et eux, que pensent-ils de vous?

Mesa : Je ne sais. Je ne m'en occupe pas. Je ne pense pas aux autres.

Ysé : Mesa! Mesa!

Mesa : Tiens, c'est vrai ! C'est donc que je ne pense qu'à moi-même? 
Ysé : Voilà que vous le découvrez? Niez que les femmes servent à quelque chose.

(p. 86)

l'aptitude du Sujet à entendre l'Autre. Cela deviendra même l'essentiel du message que, dans ses bonnes intentions, la pièce délivre. Mais dans la version de 1906, la seule véritablement autobiographique, l'explication du rôle de la femme (de l'Autre) dans l'économie du salut, qui est pourtant clairement esquissée, sera complètement recouverte par les choix qu'implique une écriture qui s'affirme précisément - nous l'avons vu - en inféodant l'Autre à l'angle de vue du Sujet.

On peut donc penser qu'en dernière analyse, aux yeux du poète vieillissant, le travail autobiographique participe en lui-même de l'« éloignement » que dans le livre d'Osée la voix prophétique déplore : «Je les ai appelés et ils se sont éloignés ». Une telle lecture place toute l'œuvre et le projet même dont elle est issue sous le coup du blâme auquel on doit finalement comprendre qu'elle se destinait à répondre, si bien que là, encore une fois, la cellule originelle se rejoue, indépassable : « Or je voulais tout donner, / Il me faut tout reprendre » (p. 79). Ce serait pour dépasser la qualité autobiographique de son drame - jugée sévèrement - que Claudel aurait remis son drame sur le métier, l'aurait repris.

Par la suite, Claudel ouvrira de façon tout à fait originale d'autres voies à l'écriture autobiographique. Au théâtre, ce seront aussi bien le drame historique "en costume " (Le Soulier de satin) que ce qu'il baptisera du nom charmant d'«extravaganza radiophonique " : la ludique Lune à la recherche d'elle-même où le dramaturge parvenu au faite de son art interroge le poète en herbe, et l'homme vieux le jeune homme qu'il a été. Dans ces œuvres, le retour sur soi et sur le passé est confié à l'ironie, au caprice de la mémoire, au lyrisme et... à l'invention.

On ne manquera pas non plus de rappeler ce que Michel Malicet, le savant éditeur de l'œuvre exégétique de Claudel, a eu à maintes reprises l'occasion d'observer au cours de son travail sur les derniers textes de Claudel: le commentaire biblique, lié fondamentalement à la personne du poète et du croyant, à sa foi et à son espérance, devient au fil des années le nouvel espace où l'écriture autobiographique trouve à se développer. En toute liberté. Même si c'est en fonction d'un tout autre projet idéologique, Claudel rejoint par là une pratique de l'écriture autobiographique qui fut aussi celle de Montaigne.

Partage de midi 


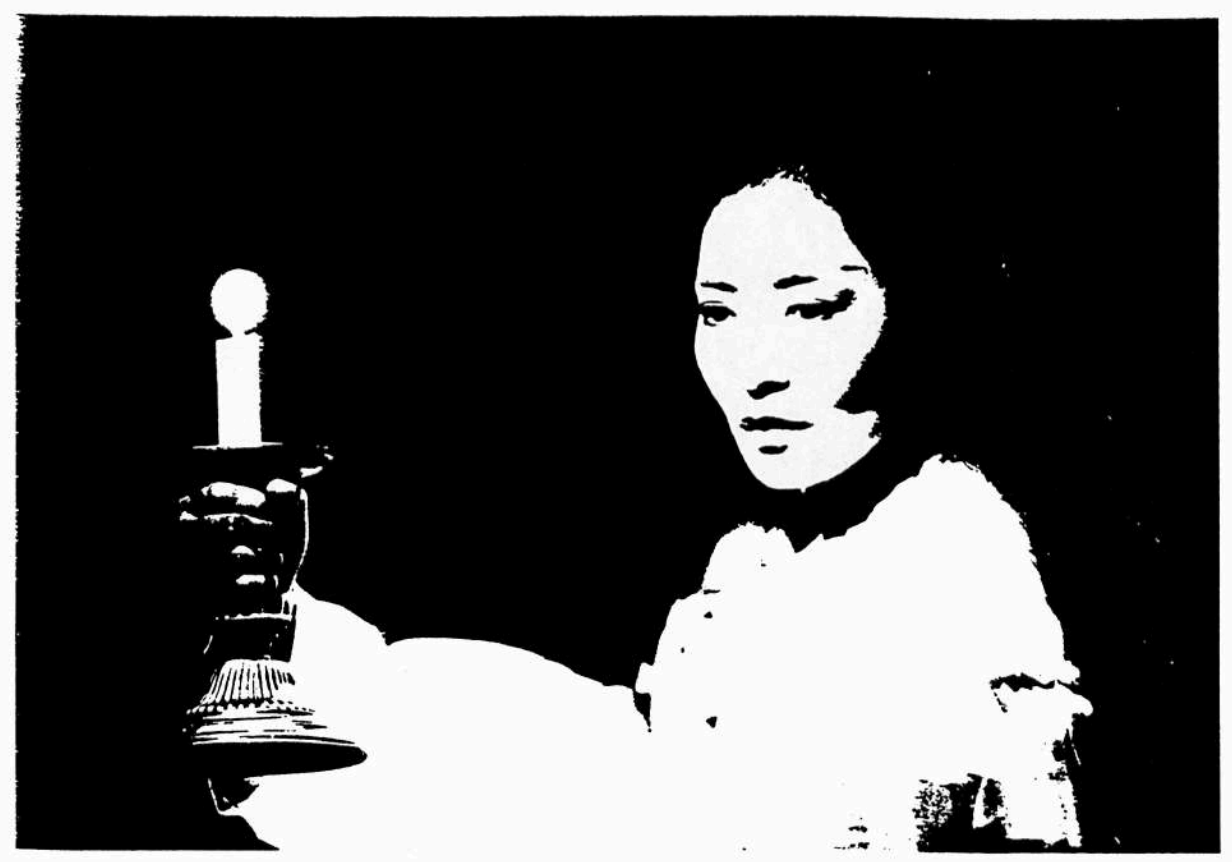

Mise en Scène de Moriaki Watanabé

Archives Centre Jacques Petit 